\title{
DIEGO DE URREA EN ITALIA
}

\author{
FERNANDO RODRÍGUEZ MEDIANO * \\ CSIC, Madrid
}

En el primero de los dos números monográficos que la revista Al-Qanțara dedicó al asunto de los libros plúmbeos del Sacromonte, Mercedes García-Arenal y yo publicamos un artículo que intentaba reconstruir la biografía de Diego Urrea ${ }^{1}$, personaje de especial relevancia en la transcripción y traducción de los plomos, y cuyo itinerario vital nos parecía en extremo interesante y ejemplificador. Quien conozca ese artículo recordará que Urrea era un italiano cautivado muy joven por los turcos; que estudió en la madrasa de Tremecén y, con el nombre de Morato Aga, llegó a ser secretario en Argel, Trípoli, Túnez y Constantinopla; que en 1589 fue hecho prisionero por unos cristianos que le llevaron a Palermo, donde quedó bajo el amparo del conde de Alba de Liste y se convirtió al cristianismo, adoptando el nombre de Diego de Urrea; que viajó, por fin, en 1591, a España, donde fue catedrático de árabe en la Universidad de Alcalá ${ }^{2}$, amigo y maestro de los Argensola, catalogador de los manuscritos árabes de El Escorial, traductor de los Plomos e intérprete-intermediario ante el sultán marroquí Muley Xeque en toda la cuestión de la entrega de Larache a los españoles. La pista de su vida se nos perdía precisamente alrededor de 1610, cuando esa negociación estaba en su apogeo. Como ya indicamos en ese momento, Diego de Urrea había expresa-

\footnotetext{
* Este artículo ha sido realizado dentro del proyecto «Falsificaciones de la historia en torno a los musulmanes de España (ss. XVI-XVII» (BHA2000-1509), cuya investigadora principal es Mercedes García-Arenal.

1 Rodríguez Mediano, Fernando y García-Arenal, Mercedes, «Diego de Urrea y algún traductor más: en torno a las versiones de los "Plomos"», Al-Qanțara, XXIII (2002), 499-516.

${ }^{2}$ Espero ocuparme, en un próximo trabajo, de la enseñanza del árabe en la Universidad de Alcalá en los ss. XVI y XVII. Baste decir, ahora, que Diego de Urrea fue encargado de la enseñanza del árabe en esa Universidad en 1953, y que fue adscrito al Colegio Trilingüe. V. Archivo Histórico Nacional, Universidades, Libro 1128, f. 53v y ss.
}

Al-Qanțara XXV, 1 (2004) 183-201 
do, hacia 1607, su intención de dirigirse a Nápoles, así que una de las posibilidades que apuntábamos para los últimos años de su vida era que, efectivamente, tras la resolución de la cuestión de Larache, hubiera podido volver a Italia. Pero esto no pasaba de ser una mera hipótesis. La razón de este segundo artículo sobre Diego de Urrea es precisamente completar algunos datos sobre los últimos años de su vida, que en el momento de la redacción de ese artículo desconocíamos, y que nos parece tienen un cierto interés.

\section{Diego de Urrea y la Accademia dei Lincei}

Fue Manuela Marín quien llamó nuestra atención sobre un texto presentado por Valentina Sagaria Rossi en la 24 Conferencia MELCOM International, celebrada en la Bibliohèque Nationale de France los días 27-29 de mayo de 2002, titulado «Le goût bibliophile de Leone Caetani et l'intérêt pour l'Orient de Federico Cesi: deux "lincei" en comparaison» ${ }^{3}$, y donde se hacía una interesante alusión a Diego de Urrea. En su artículo, V. Sagaria Rossi realiza una evocación de la figura de Giuseppe Gabrieli, padre del insigne arabista Francesco Gabrieli, bibliotecario de la Biblioteca de la Accademia Nazionale dei Lincei. La relación de G. Gabrieli con el príncipe Leone Caetani, gran bibliófilo e historiador, colector de impresos y de manuscritos, impulsor de los estudios orientales propiamente modernos en Italia, permite a V. Sagaria Rossi realizar una comparación entre su figura y la de Federico Cesi (1585-1630), semejantes en algunos aspectos, como el de estar ambos en posesión de «un espíritu pragmático, refinado y palpitante» y de «una disposición natural a animar y organizar la investigación científica». En efecto, Federico Cesi fue fundador, en 1603, de la Accademia dei Lincei, y uno de los principales protectores y mecenas de Galileo Galilei. Entre los intereses de Cesi y de los primeros lincei se contaba, en mayor o menor medida, el de la lengua árabe. De hecho, como se verá, el propio Cesi estudió árabe con un cristiano oriental, y, en los primeros momentos de la Accademia, contó entre sus miembros, por consejo del linceo napolitano Giovan Battista della Porta, con el traductor del árabe y experto en lenguas orientales Diego de Urrea

\footnotetext{
${ }^{3}$ El texto puede encontrarse en la dirección http://www.uni-bamberg.de/unibib/melcom/Sagaria\%20Rossi-1.html.
} 
Conca, que a la sazón se encontraba en Nápoles. Como el propio Cesi escribía a Galileo, Urrea podría encargarse de la traducción de textos árabes sobre matemáticas y ciencias naturales.

No es casualidad que la principal fuente de información que, a este respecto, utiliza V. Sagaria Rossi (además de su propio conocimiento de los archivos, como miembro de la Sezione Orientale de la Biblioteca Nazionale dei Lincei e Corsiniana) sean los estudios de G. Gabrieli, que exploró con gran erudición la historia de la Accademia en varios trabajos que, en lo que nos atañe, constituyen aún la principal fuente de información sobre la actividad de Diego de Urrea como miembro de la sección napolitana de los Lincei.

La nómina de las obras que formaban parte de la primera Biblioteca Lincea es muestra del interés de Federico Cesi por los estudios orientales ${ }^{4}$. Sin duda debió compartir ese interés con uno de sus grandes amigos, el filósofo napolitano Giovan Battista della Porta, a quien conoció durante su primer, y quizás único, viaje a Nápoles ${ }^{5}$. La relación entre ambos se mantuvo durante tiempo, hasta que finalmente Giovan Battista della Porta se adhirió a la Accademia del Lincei, fundada por Cesi. Fue precisamente en el curso de esta relación cuando Della Porta comenzó a informar a Cesi de la llegada a Nápoles de Diego de Urrea, cuyo conocimiento del árabe podía ser útil para sus propios intereses científicos. Así, el 26 de diciembre de 1611, Giovan Battista della Porta escribía una carta a Federico Cesi, que incluía el siguiente párrafo: «Scrissi a V.S. che in Napoli haveremo un huomo secretario del Re nostro delle lingue, et è dell'Araba peritissimo, se V.S. volesse sportare in italiano alcun libro, io pigliare questo pensiero" ${ }^{6}$.

El propio Diego de Urrea da cuenta, en una carta enviada al cardenal Federico Borromeo, el 4 de octubre de 1611, de su llegada a Nápoles y de su intención de colaborar con éste en la adquisición de libros árabes y en la traducción de algunos textos. Como se sabe, el cardenal Borromeo tuvo un extraordinario interés por la ciencia, el conocimiento y la bibliografía, que le llevó a fundar en 1609 la biblio-

\footnotetext{
${ }^{4}$ Gabrieli, G., «I primi accademici lincei e gli studi orientali», Contributi alla storia della Accademia dei Lincei, Roma, 1989, I, 331-345.

5 Gabrieli, G., «Giovan Battista della Porta linceo da documenti per gran parte inediti», id., 635-685.

${ }^{6} I d ., 648$.
} 
teca Ambrosiana de Milán. Fue precisamente este interés bibliófilo y científico del cardenal Borromeo lo que explica la carta de Urrea que, en ciertos aspectos, ofrece un gran interés. Su texto es el que sigue, según la edición de $\mathrm{G}$. Gabrieli ${ }^{7}$, que corrijo solamente en algunos lugares donde el error me parece evidente:

[...] He recevido la carta de V.S. Illma de los siete de setiembre, del qual he conecido de mucho mas la grandeza del [sic] V.S. Illma, accompañada de tanta humiltad en haverse diñado escrever con tanto regalo a tan afficionadissimo criado ${ }^{8}$ suyo. Haurà cosa de dos meses que lleguè a Napoles, donde pienso estar ${ }^{9} \mathrm{de}$ assiento ${ }^{10}$ ò almeno doss años, y assi entodo estoy rendido a la voluntad de V.S. Illma en mandarme en todo quanto fuere servido.

Me huelgo mucho deque V.S. Illma aya sido servido mandar enviar a levante por libros, y principalmente por el Camus, que es uno de los mejores y mas provechoso libro [sic] que ay en aquellas provincias; que si lo uviera savido huviera enviado una memoria de los libros que avian de buscar.

Me ofresco a la traducion del dicho Camus, que sera trabajo muy provechoso para la yglesia de Dios y estirpacion de la secta mahometana; que tengo per [sic] cosa infalibile que si en la Christianitad huviera theologos doctos en la lingua arabica, no huviera prevalecido tanto la mention [?] ${ }^{11} \mathrm{y}$ bestial secta de Mahoma. Pienso que el Camus lo tiene el s. Duque de Florensia en la libraria que dicen Medicea. Y por via ${ }^{12}$ de Malta, y dunque de Florensia, podría V.S. Ill.ma haver muchos libros.

Y por no faltar un momento del servicio de la Mag. del Rey Catholico mio senor (aquien estoy dedicado servir y morir), huviera partido a besar ${ }^{13}$ los pies y ofrecerme actual(men)te en lo que fuera servido mandarme. como de nuevo me ofresco.

Aqui he encontrado un criado de V.S. Ill.ma llamado el doctor Francesco Placa, y me ha referido la grandeza de la libreria de V.S. Ill.ma, y es muy afficionado a V.S. Illma y procura que todos accedan al servicio [...].

En efecto, como el propio Gabrieli indica, el caballero milanés Francesco Piazza había sido enviado por Borromeo a Nápoles con el encargo de buscar y adquirir libros raros. Allí había encontrado a

7 G. Gabrieli, «Federico Borromeo e gli accademici lincei», id., II, 1464-1486. La carta se encuentra en 1483-4.

${ }^{8}$ En la edición de Gabrieli, «chiado», que corrijo.

9 En la edición de Gabrieli, «esser», que corrijo.

${ }^{10}$ En la edición de Gabrieli, «assienso», que corrijo.

11 Sic en la edición de Gabrieli. ¿Debe corregirse, quizás, por «maldita»?

12 En la edición de Gabrieli, «mia», que corrijo.

13 En la edición de Gabrieli, «basar», que corrijo. 
Urrea, y admirado en él «il discorso, la prudenza, la pratica, l'humanità et gentilezza, il sapere [...]», tal como escribió al cardenal Borromeo ${ }^{14}$ en una carta en la que añadía: «Io so che in casa di Gio. B. [Della Porta] un giorno in una corona di virtuosi fece restar meravigliati tutti et consolati insieme con una lettione que fece, ispiegando alcuni luoghi d'una geografia araba [...], traducendola in spagnolo con molta eruditione et con mostra chiarissima della prontezza che possiede tal lingua» ${ }^{15}$. A partir, pues, de este encuentro con Piazza, Urrea ofreció sus servicios al Cardenal Borromeo para ayudarle en la adquisición de libros árabes, $\mathrm{y}$, más concretamente, en la traducción de al-Qāmūs al-muhịt de al-Fīrūzābādī, libro que, al parecer, había encargado buscar en Oriente. La Biblioteca Ambrosiana cuenta, en efecto, con al menos dos copias del Qämūs, una de las cuales, por cierto, es obra del inglés David Colville, quien la realizó en 1623 a partir de los ejemplares existentes en la Biblioteca de El Escorial ${ }^{16}$.

Sea como fuere, el ofrecimiento de colaboración con el Cardenal Borromeo no dejó ninguna traza aparente. Urrea afianzó más bien sus relaciones con el ambiente cultural napolitano, y de manera especial con el círculo de Giovan Battista della Porta, quien esperaba poder emplear con provecho las cualidades de Urrea como arabista para realizar traducciones de textos de interés científico: interés que, como se ha visto, ligaba a Della Porta con Federico Cesi. De esta forma, cuando a principios de 1612 se instauró de manera oficial una Colonia Lincea en Nápoles patrocinada por G.B. Della Porta, Diego de Urrea fue uno de sus cuatro miembros, junto con Filesio Constanzo della Porta, Nicola Antonio Stelliola y Fabio Colonna ${ }^{17}$. Así, el 2 de febrero de ese año, Diego de Urrea firmaba su adscripción a la Accademia con el siguiente texto escrito en árabe y en latín ${ }^{18}$ :

${ }^{14}$ G. Gabrieli, «Federico Borromeo e gli accademici lincei», 1470.

15 Id., 1470-1.

${ }^{16}$ Löfgren, O. y Traini, R., Catalogue of the Arabic Manuscripts in the Biblioteca Ambrosiana, Vicenza, 1975, I, mss. LXXI, CXXXI y CCCLIII-W.

$17 \mathrm{G}$. Gabrieli evoca este momento en varios lugares de sus trabajos sobre la Accademia. V., por ejemplo, «Verbali delle adunanze e cronaca della prima Accademia Lincea (1603-1630)», Contributi, 497-550; 517. V. también Minieri Riccio, Camillo, Accademie fiorite nella cittè di Napoli, Nápoles, 1879, 70-1.

18 Gabrieli, G., Contributi, II, Tav. XVI; Il carteggio linceo, Roma, 1996, 201. 


$$
\begin{aligned}
& \text { ديقوا ني اوريه قونقه كاتب السرّ للسلطان الاعظم سلطان / اسبانية في مسايل افريقية وآسيّة }
\end{aligned}
$$

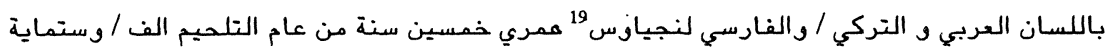

$$
\begin{aligned}
& \text { راثنى عشر ثاني يوم شهر فبرارس ومي في نابلس / بخط يدي }
\end{aligned}
$$

Ego Didacus de Urrea Conca Joannis Aloy / sij filius lynçeus neapolitanus a se / cretis epistolarum Africae et Asiae in / lingua arabica turcica et persica Regnis / ispaniarum aetatis maee [sic] anno 50 salutis / 1612 die 2 februariy neapoli manu mea / scripsi

$\mathrm{Al}$ día siguiente de redactar esta adscripción, Diego de Urrea enviaba una carta a Federico Cesi en árabe ${ }^{21}$, en la que le hacía saber cuán honrado se sentía de haber sido aceptado en tan sapientísima sociedad, de la que era indigno por su poco saber, pero a la que se ofrecía a ayudar en todo lo que estuviese a su alcance. El propio Cesi, al informar a Galileo de las nuevas incorporaciones napolitanas a la Accademia en una carta de 17 de marzo de 208, le hablaba de «il sr. Don Diego de Urrea, cavalier nobile et di dottrina non ordinaria, poichè, oltre la filosofia et buona cognition d'altre scienze, ha compitamente la lingua arabica persiana et turchesca; fu prima secretario del Re di Fez, hora è di quello di Spagna, di dette lingue, provigionato di cinque mila scudi l'anno, come mi scrivono", al tiempo que le remitía la carta en árabe que el propio Urrea le había dirigido a él ${ }^{22}$. El hecho de que Urrea escribiese su carta en árabe es una muestra de que el interés de este grupo por esa lengua no se limitaba a la bibliofilia, o a la búsqueda de manuscritos de interés científico, sino que impulsó a varios de ellos a aprender el árabe, como se verá más adelante. Como señala Gabrieli, algunos indicios muestran que muchos Lincei poseían un conocimiento del árabe bastante estimable: así, cuando Federico Cesi pedía a un amigo que intentase que Urrea respondiese a todos los que le habían escrito, «e se vole in Arabico» ${ }^{23}$.

¿Cuáles fueron los trabajos de traducción que los académicos encargaron a su nuevo compañero? El 2 de junio de 1612, Giovan Battista della Porta escribía una carta a Cesi en la que le decía: «Mi avisi V.S. se vuole che le mandi quelle carte arabiche; e se verrà il libro,

${ }^{19}$ Lectura probable. Se trata de una arabización del término «linçeus».

20 En el original, فلوارس.

21 Gabrieli, G., Carteggio, 201; reproducción del original en Contributi, II, Tav. VII.

${ }^{22}$ Gabrieli, G., Carteggio, 209.

${ }^{23}$ Carta de Federico Cesi a Francesco Stelluti, mediados de abril de 1613, id., 344. 
sarà subito tradutto, che il S.r Diego Urrea sta avidissimo di servire a V.S., e mostrarseli grato» ${ }^{24}$. G. Gabrieli confiesa no saber a qué libros ni a qué cartas se refería Della Porta en este párrafo, aunque en la correspondencia lincea hay alguna otra referencia a libros árabes cuya traducción se requería ${ }^{25}$. Las alusiones son suficientemente vagas como para no tener certeza de cuáles son las obras en cuestión. Gabrieli ha revelado, sin embargo, una alusión más concreta a un libro que el círculo de Cesi estaba interesado en traducir ${ }^{26}$. En una carta de 15 de febrero de 1614, Cesi informaba a Galileo de la muerte de Giovanni Battista Raimondi, ilustre orientalista y tipógrafo, impulsor de la fundación de la Tipografia Medicea Orientale en $1584{ }^{27}$. La muerte de Raimondi había paralizado su proyecto de imprimir algunos de los manuscritos árabes que custodiaba. Cesi añadía: «Io haverei desiderio particolare che sei o otto volumi di cose naturali e matematiche, che non habbiamo in latino, fossero tradotti e si stampassero [...]. Parò mi farà gratia V.S. d'intendere che mente habbia S.A. in queste cose del Raimondi; e se le parrà ottenibile, veda d'impetrare che di questi volumi partocolari si potesse far copia, a fine che fossero tradotti e stampati, dedicati a S.A. come conviene, ché noi habbiamo il S.r Don Diego d'Urrea che lo farebbe benissimo»' ${ }^{28}$. Uno de esos manuscritos que quedaron en la biblioteca de Raimondi, y cuya impresión interesaba a Galileo y a Cesi, era la versión árabe de las Cónicas de Apolonio de Pérgamo, de cuyos últimos libros no existía versión latina ${ }^{29}$. He aquí, pues, un proyecto concreto en el que Cesi y Galileo pensaron emplear la destreza arabista de Diego de Urrea. Lo cierto es, sin embargo, que tal empresa no se llevó a cabo, y que Urrea murió al poco tiempo, quizás en 1615, aunque no queda constancia documental cierta al respec-

${ }^{24}$ Gabrieli, G., «Giovan Battista della Porta linceo da documenti per gran parte inediti», 654; Carteggio, 233.

${ }^{25}$ Carta de Federico Cesi a Francesco Stelluti, 5 de julio de 1612, Gabrieli, G., Carteggio, 247.

${ }_{26}$ Véase la argumentación de Gabrieli en «I primi accademici lincei», 338 y ss.

27 Sobre esta Tipografia, y sobre Raimondi, v. Balagna, Josée, L'imprimerie arabe en Occident (XVIe, XVIIe et XVIIIe siècles), París, 1984, 35 y ss. No he podido consultar Tinto, Alberto, La tipografia medicea orientale, Lucca, 1987.

${ }^{28}$ Gabrieli, G., «I primi accademici lincei», 338 y ss.; Carteggio, 417.

29 Sobre Apolonio de Pérgamo en la cultura árabe, v. Plessner, M., EF ${ }^{2}$, s.v. «Balīnūs», donde se podrán encontrar las referencias bibliográficas oportunas. La primera edición moderna de los libros V-VII de las Cónicas fue una traducción latina del texto árabe realizada por Abraham Ecchellensis y Giacomo Alfonso Borelli, Florencia, 1661 (Sarton, G., Introduction to the History of Science, Baltimore, 1927, I, 174). 
to, sino simple conjetura. Sea como fuere, no quedó rastro de ninguno de los proyectos citados.

\section{Diego de Urrea y el Conde de Lemos}

¿Por qué había Diego de Urrea dirigido finalmente sus pasos hacia Nápoles? Uno de los acontecimientos sociales y políticos más sonados en España en 1610 fue la incorporación de D. Pedro Fernández de Castro, Conde de Lemos, a su cargo de Virrey de Nápoles ${ }^{30}$. El puesto era muy importante, y el nombre de Lemos había surgido ya varios años antes como uno de los más idóneos para ocuparlo, cuando su padre D. Fernando lo dejó vacante al morir en 1601, y otra vez, después, en 1606. Ello significó que durante todo ese tiempo se crease una gran expectativa en torno al virreinato de Lemos, no sólo porque Nápoles era uno de los dominios más importantes y significados de la monarquía hispánica en Europa, sino por el propio carácter del conde, sin duda el más grande de los mecenas literarios españoles de su tiempo. Así lo atestigua, por ejemplo, el elevado número de escritores que le dedicaron sus libros, incluido el propio Cervantes con la segunda parte del Quijote ${ }^{31}$. La perspectiva de la constitución en Nápoles de una gran corte literaria en torno al nuevo virrey animó la ambición y las esperanzas de muchos escritores que pensaron podrían acompañar al Conde en su inminente destino. Así, durante algún tiempo, menudearon en la Corte los rumores, las maniobras, las intrigas literarias, $\mathrm{y}$, finalmente, en buen número de casos, la frustración, la resignación más o menos irónica, tal vez la maledicencia de quienes no alcanzaron a cumplir su ilusión napolitana. Entre los aspirantes a acompañar a Lemos se cuentan nombres tan ilustres como los de Cervantes o Góngora, en cuya obra pueden encontrarse alusiones directas a esta aventura.

En todo este inquieto episodio previo a la partida del Conde de Lemos para Nápoles desempeñaron un papel importantísimo las per-

\footnotetext{
30 Para lo que sigue, v. el estudio clásico de Green, Otis H., «The Literary Court of the Conde de Lemos at Naples, 1610-1616», Hispanic Review, I-4 (oct. 1933), 290-308. V. también Fernández Murga, F., «El Conde de Lemos, virrey-mecenas de Nápoles», Annali dell'Istituto Universitario Orientale. Sezione Romanza, IV, 1 (ene., 1962), 5-27.

${ }_{11}$ Simón Díaz, J., «Libros dedicados al gran Conde de Lemos», El Museo de Pontevedra, XLIV (1990), 247-266.
} 
sonas de su entorno más inmediato, encargados de la gestión sus asuntos. Entre ellos destacaban sobre todos Bartolomé y Lupercio Leonardo de Argensola, y especialmente este último, que era secretario del conde desde 1608, y que tenía capacidad de decisión directa sobre la elección del personal a su servicio. Las quejas de algunos escritores hacia el comportamiento de Lupercio en ese momento son buena muestra de su importancia a la hora de confeccionar la corte de Lemos, en la que, según algunos, más que poetas, figuraban «señores amigos dellos» (es decir, de los Argensola) ${ }^{32}$. En efecto, el escritor más destacado que acompañó al conde a Nápoles fue Antonio Mira de Amescua. Los demás (Gabriel de Barrionuevo, Antonio de Laredo, Francisco de Ortigosa y Gabriel Leonardo de Albión, hijo de Argensola) no estaban a la altura literaria de otros pretendientes ${ }^{33}$.

Sin embargo, el papel fundamental de Argensola como secretario de Lemos nos permite suponer que la razón por la que Diego de Urrea fue a Nápoles fue precisamente su relación privilegiada con él, puesto que sabemos que había sido su profesor de árabe, y que ambos mantuvieron una amistad continuada, como demuestra el epistolario del poeta aragonés ${ }^{34}$.

El virreinato del Conde de Lemos en Nápoles fue uno de los momentos más destacados de la presencia española en esa ciudad. Su política se dejó sentir en todos los órdenes y, también, por supuesto, en el cultural, como corresponde a quien destacaba como mecenas literario. Una de las actividades que Lemos impulsó en Nápoles fue, precisamente, la fundación, a instancias de Giovambattista Manso, de una academia literaria, la Accademia degli Oziosi, que celebró su primera reunión el 13 de mayo de $1611^{35}$. Las actividades de la Accademia

32 Green, O. H., «The Literary Court», 295. En un irónico y elegante soneto cuyo tema es en parte la partida a Nápoles del Conde de Lemos, Góngora escribió «como sobran tan doctos españoles / a ninguno ofrecí la musa mía», refiriéndose a la excesiva influencia de los Argensola sobre el conde, id., 294.

33 Id., 295.

34 Rodríguez Mediano, F. y García-Arenal, M. «Diego de Urrea», passim. El propio Conde de Lemos alude a Diego de Urrea en una carta dirigida al Rey desde Nápoles el 6 de diciembre de 1612: «También quedo con cuydado de pagar y acomodar a Diego de Urrea sabiendo la merced que Vuestra Magestad le hace», en Pardo de Guevara y Valdés, E., con la colaboración de Rodríguez Suárez, P. y Barral Rivadulla, D., Don Pedro Fernández de Castro VII conde de Lemos (1576-1662). II. Colección documental, Santiago de Compostela, 1997, 203.

${ }^{35}$ A los trabajos citados pueden añadirse otros, como Cirillo, Teresa, «Letterati italiani e spagnoli nell'Accademia degli Oziosi», en Bosse, Monika y Stoll, André (coor.), 
intentaban integrar a la sociedad literaria hispano-napolitana dentro del sistema cortesano, y la selección de sus miembros y la instauración de las normas de comportamiento estaban determinadas, en buena medida, por este afán. Así, en los debates académicos primaban las cuestiones literarias, científicas o filosóficas, con exclusión explícita de temas políticos y teológicos, y con un severo control sobre las cuestiones debatidas. En este sentido, la actividad de la Accademia degli Oziosi derivó en realidad hacia una práctica lúdico-erudita, en correspondencia con «la función social y mundana de la fiesta y del juego» ${ }^{36}$ : los académicos hablaban exclusivamente en verso, $\mathrm{y}$, más que profundizar en las cuestiones propuestas, los debates se convertían en justas donde mostrar el «ingenio» ${ }^{37}$. Es muy famoso el fragmento de Diego Duque de Estrada donde se describe una sesión de la Accademia en el curso de la cual se improvisó una comedia sobre Eurídice, en la que Bartolomé Leonardo de Argensola («hombre graciosísimo, viejo y sin dientes») interpretaba a Proserpina, «vestido de dueña» e improvisando versos como «Yo soy la Proserpina; ésta, la morada / del horrible rabioso cancerbero, / que me quiere morder por el trasero" ${ }^{38}$.

Este ambiente brillante e ingenioso de la Accademia degli Oziosi (en cuyas sesiones llegó a participar el Conde de Villamediana y, como algunos han sugerido, quizás erróneamente, el propio Francisco de Quevedo durante su estancia napolitana) era reflejo, como se ha dicho, de la concepción orgánica que de la actividad cultural tenía el Conde de Lemos, que pretendía unificar los diversos componentes de las instituciones intelectuales napolitanas dentro de un proyecto global de imposición de la hegemonía cultural y política española ${ }^{39}$. Desde este punto de vista, la fundación o adscripción a una u otra academia letrada tenía implicaciones que iban más allá de un simple encuadramiento intelectual. Así, por ejemplo, Federico Cesi tuvo una

Napoli viceregno spagnolo. Una capitale della cultura alle origini dell'Europa moderna (sec. XVI-XVII), 2 vols., Nápoles, 2001, II, 169-181. Para un fino análisis de los estatutos de la Accademia, v. Ivo Comparato, Vittor, «Società civile e società letteraria nel primo seicento: 1'Accademia degli Oziosi», Quaderni Storici, 23 (mayo-agosto 1973), 359-388.

${ }^{36}$ Para todas estas cuestiones, v. Cirillo, T., «Letterati», 177.

37 Green, O. H., «The Literary Court», 305-7.

38 Duque de Estrada, D., Comentarios del desengañado de si mismo. Vida del mismo autor, ed. Henry Ettinghausen, Madrid, 1983, 195-6.

39 Olmi, Giuseppe, «La colonia lincea di Napoli», en Lomonaco, Fabrizio y Torrini, Maurizio (coor.), Galileo e Napoli. Atti del convegno Galileo e Napoli. Napoli, 12-14 aprile 1984, Nápoles, 1987, 23-58, 37. 
preocupación especial por el papel que debía desempeñar la colonia napolitana de los Lincei bajo la dirección de G.B. Della Porta. En la concepción de Cesi, el funcionamiento de su Accademia suponía una dedicación ascética a la ciencia y al conocimiento, que proponía a sus miembros un auténtico modelo de vida. Así, el distanciamiento voluntario de la sociedad romana de su tiempo, y la renuencia a inscribirse en sus estructuras sociales o universitarias, apuntaba sobre todo a un proyecto de alcance europeo, en el que distintos grupos de intelectuales organizados de un modo parejo podrían recomponer la unidad del mundo a través de una similar concepción del conocimiento y de la investigación científica ${ }^{40}$. Parece evidente que esta concepción de la actividad de su propia academia podía chocar, en Nápoles, con el proyecto cultural del Conde de Lemos. Por eso, Cesi se esforzó en presentar al Conde la actividad de los Lincei napolitanos de una forma sumamente humilde, subrayando su lealtad hacia el Virrey y sus propósitos estrictamente científicos y matemáticos, buscando preservar, en cierto modo, su autonomía. En este mismo marco cabe entender, quizás, la elección de Diego de Urrea como académico de los Lincei, contraviniendo los propios estatutos de la Accademia, que sólo admitían a personas con preparación específica en el campo de las ciencias naturales o de las matemáticas. Dicho de otro modo: Urrea podía representar para Cesi no sólo la posibilidad de traducir textos árabes de contenido científico, sino una maniobra más o menos oportunista para complacer a G.B. Della Porta, la persona clave del Linceo napolitano, y quien podría dirigirlo y asegurar su supervivencia en un contexto que podía ser problemático ${ }^{41}$. Sin embargo, esta contradicción se reveló difícilmente superable: el propio G.B. Della Porta se adhirió desde el primer momento a la Accademia degli Oziosi, y fue uno de sus miembros más activos; bajo su dirección, además,

40 Id., 23-6. No conviene, sin embargo, realizar una separación demasiado neta entre una academia filosófica y científica y el mundo cortesano romano. Cesi pertenecía a la alta nobleza de Roma, y aunque desdeñoso por el aparato cortesano y los modos agresivos de los nuevos advenedizos, conocía bien la sutileza de la política romana. Su desapego del mundo cortesano se tradujo en el carácter mismo de su Accademia, que él concebía más bien como una sociedad jerárquica y aristocrática, organizada en torno a su persona, representante de un ethos feudal y caballeresco: más que a un monasterio, la Accademia se parecería a una orden militar. V. Biagioli, Mario, Galileo Courtier. The Practice of Science in the Culture of Absolutism, Chicago-Londres, 1993, 292 y ss.

41 Todo este párrafo reproduce la argumentación de Olmi, «La colonia lincea di Napoli», 38 y passim. 
se potenció el aspecto mas superficial, público y social de la colonia napolitana de los Lincei, con un cierto abandono del proyecto inicial de Cesi ${ }^{42}$. Diego de Urrea se encontró, pues, en medio de una situación ambigua, que no permitía realmente la puesta en marcha de ningún proyecto intelectual de alcance, pero también apasionante, en un momento crucial de la presencia española en Italia. No he encontrado la prueba de que Urrea perteneciese también, como Della Porta, a la Accademia degli Oziosi, aunque me parece lo más probable. Sin embargo, en la lista más extensa de oziosi que he podido consultar no figura su nombre ${ }^{43}$. Sea como fuere, toda la aventura napolitana de nuestros personajes fue bastante fugaz: Leonardo Lupercio de Argensola murió en 1613, y Diego de Urrea, probablemente, en 1615.

\section{Italia y los estudios árabes: Marcos Dobelio}

La presencia de un buen conocedor del árabe como Diego de Urrea en el ambiente italiano de comienzos del s. XVII es un hecho notable, pero no excepcional. El interés por el árabe y por los libros en árabe era grande entre la élite intelectual italiana, como demuestran los propios contactos de Urrea con G.B. Della Porta, Federico Cesi o el Cardenal Borromeo, producto del afán por compilar libros y conocimientos científicos. También se ha visto cómo Urrea podía mantener correspondencia epistolar en árabe con algunos lincei, lo que muestra una competencia notable en esa lengua entre algunos académicos. La de la circulación de libros y manuscritos árabes en Italia, y la del conocimiento y aprendizaje del árabe, son historias complejas, que exceden infinitamente el alcance del presente texto. Sin embargo, pueden señalarse aquí un par de líneas que, teniendo relación con Urrea, no carecen de interés.

Un año después de la (probable) muerte de Urrea, el doctor Mario Schipano era propuesto para su adscripción a la Accademia dei Lincei. G. Gabrieli cree que esta propuesta se explicaba por la voluntad de Federico Cesi de contar entre los miembros de la Accademia a personas expertas en árabe y en cosas orientales en general, y que fuesen capaces, de

42 Id. 45-7.

${ }^{43}$ Minieri Riccio, C., Accademie, 83 y ss. En la p. 85, n. 5, figura, sin embargo, un tal «Concubletto». ¿Se trata, quizás, de una deformación humorística (tan del gusto de los académicos) de «Conca»? 
manera más concreta, de traducir la obra de Apolonio de Pérgamo ${ }^{44}$. Schipano era un médico calabrés que, desde joven, había entrado en contacto con G.B. Della Porta y con el círculo de los lincei napolitanos. Además de su actividad como profesor en el Studio de Nápoles y, después, como protomédico en la Casa de la Santissima Annunziata (institución eclesiástica asistencial de Nápoles), era poeta aficionado y buen conocedor del griego y del árabe. Gabrieli supone, con cierto fundamento, que su profesor de árabe fue Diego de Urrea, con quien debió coincidir en casa de Della Porta desde $1611^{45}$. Pero si el nombre de Mario Schipano mereció alguna fama póstuma fue por su relación con el célebre viajero italiano Pietro della Valle, íntimo amigo y correspondiente suyo. Della Valle narró su viaje a Oriente en cartas que dirigía a Schipano, y que luego recogió en un volumen dedicado a su memoria ${ }^{46}$. Más aún: el viaje oriental de Della Valle le sirvió para recopilar libros y manuscritos, que luego pasaron a engrosar la buena biblioteca oriental de Schipano. No parece casual que uno de los libros que éste le encargó buscar en Oriente fuese precisamente el Qāmūs de al-Firūzābādī ${ }^{47}$. En todo caso, el propio Della Valle pudo conocer a Diego de Urrea cuando frecuentaba la casa de G.B. Della Porta en $1612^{48}$, en un momento especialmente importante de su vida, cuando la relación con Schipano, Della Porta y otros le hizo concebir el proyecto de su viaje oriental. Para terminar con el perfil de Schipano, baste añadir que fue también, entre otras cosas, miembro de la Accademia degli Oziosi ${ }^{49}$.

Como se ha visto, también Federico Cesi sabía el suficiente árabe como para poder leer la carta que Diego de Urrea le escribió en esa lengua en 1612. Existe evidencia documental, rescatada también por G.

\footnotetext{
${ }^{44}$ Gabrieli, G., «I primi accademici lincei», 342-344; «Il linceo di Napoli. Lincei e linceabili napoletani amici e corrispondenti della vecchia Accademia dei Lincei nel Mezzogiorno d'Italia», Contributi, II, 1497-1548, 1523-1529.

45 Gabrieli, G., «Il linceo di Napoli», 1519.

46 Rossi, Ettore, «Pietro della Valle orientalista romano (1586-1652)», Oriente Moderno, XXXIII, n. 1 (enero 1953), 49-64; Salvante, Raffaella, Il «Pellegrino» in Oriente. La Turchia di Pietro della Valle (1614-1617), Florencia, 1997; Gurney, J. D., «Pietro della Valle: The Limits of Perception», Bulletin of the School of Oriental and African Studies, XLIX-1 (1986), 103-116. En estos textos pueden ampliarse las noticias bibliográficas sobre Della Valle, además de las referencias concretas sobre su relación con Schipano y Diego de Urrea.

47 Gabrieli, G., «Il linceo di Napoli», 1527.

48 Salvante, R., Il «Pellegrino», 21.

49 Además de en los lugares citados, puede encontrarse la noticia en Minieri Riccio, C., Accademie, 85.
} 
Gabrieli, del temprano interés de Cesi por el estudio del árabe. En una carta enviada a Francesco Stelluti el 17 de julio de 1604, Cesi le recomienda «ruminare anco et esercitar le lettioni Arabiche per poter acquistar tutta la lingua, nella quale trovo esser scritte bellissime scienze, quale in nissun altra lingua si trovano». Más adelante añadía «attendo agli studij col maggior fervore ch'habbia mai fatto; i miei maestri sono per il più $\mathrm{i}$ libri, di voce il $\mathrm{P}$. Alessandro et l'Arabicho» ${ }^{50}$. De esta alusión de Cesi, Gabrieli deduce que este "Arabicho» a quien presenta como su profesor de árabe fue un sirio o maronita, de los que entonces estaban en Roma; quizás, añade Gabrieli, Marco Dobelio o al-Du'ābilī («Rom. Gymnasio linguae arabicae profess.» ${ }^{51}$ ), que había compuesto el epigrama árabe en honor de G.B. Della Porta que figuraba al frente de una obra de éste, De aëris transmutationibus, Roma, $1610^{52}$.

Marcos Dobelio es un nombre conocido para quienes se ocupan de la historia de los Libros Plúmbeos y de su traducción. Algunos datos sobre su vida y actividad en Roma y en España han sido ya estudiados ${ }^{53}$. Cristiano de origen kurdo, fue profesor de árabe en la Sapienza de Roma entre 1606 y 1610. Levi della Vida cita una carta en árabe (conservada en la Bodleian Library) que el propio Dobelio escribió a Isacco Casaubon el 24 de julio de 1613, ya desde España, en la que narra algunos episodios de su vida: que había llegado a España en 1610, llamado por el confesor del rey y el Cardenal de Toledo, Bernardo Rojas Sandoval, para que tradujese los libros plúmbeos descubiertos en Granada; que, por esa oferta, había dejado «mi escuela, mi oficio como secretario del Papa (kitäbatífi maktabat al-pāpā) y como traductor de las iglesias orientales, mi salario y mis libros»; que, cuando había descubierto y defendido la falsedad de los libros, el arzobispo de Granada, airado, le hizo suspender el trabajo como traductor; que, por esta razón, se encontraba en ese momento sosteniendo una causa para que le fuese pagado lo que se le había prometido; que, finalmente, estaba dispuesto a renunciar a un puesto de intérprete que se le había ofrecido en España por 600 escudos, para dirigirse a Ingla-

${ }^{50}$ Gabrieli, G., «I primi accademici lincei», 344.

51 Gabrieli, G., Il Carteggio, 39, n. 2.

52 Gabrieli, G., «I primi accademici lincei», 341.

53 Levi della Vida, Giorgio, Ricerche sulla formazione del più antico fondo dei manoscritti orientali della Biblioteca Vaticana, Ciudad del Vaticano, 1939, 280 y ss; Morocho, Gaspar, «Estudio Introductorio» a El discurso sobre el pergamino y las láminas de Granada, en Valencia, Pedro de, Obras Completas, León, 1999, IV, 307 y ss. 
terra ${ }^{54}$. Este proyectado viaje a Inglaterra, sin embargo, no se produjo, y Dobelio continuó en España, ejerciendo una actividad que ha estudiado Gaspar Morocho con bastante detenimiento. Entre otras informaciones sobre Dobelio, Levi della Vida ha dejado constancia de su profusa actividad en la Biblioteca Vaticana, y de algún otro dato interesante: Casiri, por ejemplo, cuenta que el historiador y diplomático italiano Antonino d'Amico había conseguido de Dobelio la traducción latina que éste había realizado de los pasajes del Mujtasar ta'rỉj al-bašar de Abū l-Fidā relativos a Sicilia, y que luego fueron traducidos al italiano y publicados por Agostino Inveges; añade Casiri, además, que Campomanes poseía un manuscrito con la sinopsis de esa misma obra, realizado también por Marcos Dobelio Citerone ${ }^{55}$. Este dato, en todo caso, permite confirmar que el documento que publicamos en nuestro anterior artículo sobre Diego de Urrea, referido a un traductor de los Plomos llamado «Marco Obelio Citaroni» ${ }^{56}$, tiene en realidad como protagonista a este Marcos Dobelio. La importancia del papel de éste en toda la cuestión de los Plomos ha sido puesta de relieve por G. Morocho, quien sugiere que su perdido Discurso [...] sobre las Láminas «podría ser uno de los monumentos de la Orientalística española, durante la primera mitad del s. XVII» ${ }^{57}$. Con toda seguridad, el trabajo que actualmente realiza Maylene Cotto Andino sobre la figura de Marcos Dobelio nos permitirá conocer con mayor fundamento el auténtico alcance de este apasionante episodio, tan importante para la historia de los estudios árabes en España.

\section{Pedro de Castro busca traductores en Italia}

Habría que añadir, además, que la llegada de Dobelio a España está lejos de ser un hecho casual. En su incansable y obsesiva labor en defensa de los Plomos, el arzobispo d. Pedro de Castro movilizó una extraordinaria energía para buscar por doquier personas capaces de tradu-

54 Levi della Vida, G., Ricerche, 282-3.

55 Casiri, M., Bibliotheca Arabico-Hispana Escurialensis, reproducción de la ed. de 1760-70, Osnabrück, II, 15; Levi della Vida, G., Ricerche, 281, quien añade que d'Amico visitó España a fines de 1618, ocasión en la que probablemente encontró a Dobelio.

56 Rodríguez Mediano, F. y García-Arenal, M., «Diego de Urrea», 513-515. El documento en cuestión está en el Archivo General de Simancas, sección Estado, Leg. 2645.

57 Morocho, G., «Estudio Introductorio», 312. 
cir los Libros Plúmbeos del Sacromonte. Sabemos que su búsqueda no se limitó a España, sino que se extendió al Norte de África, los Países Bajos, Italia... De hecho, Dobelio fue en principio un nombre más en la lista de expertos arabistas que, desde Italia, podían haber viajado a España para encargarse de esa tarea. Un documento del Archivo de la Abadía del Sacromonte, sin firma y sin fecha (aunque puede situarse en 1608), consiste en una lista enviada desde Italia, donde se consideran los nombres de los posibles candidatos y su disponibilidad ${ }^{58}$. Algunos de los nombres de esa lista son bien conocidos. El primero es Giovan Battista Vecchietti, "gentil'huomo ferentino, scienciato, prattico per il mondo, versatisimo nella lingua Persiana, Arabica, Torchesca, et latina et in altre». Según el documento, el señor Vecchietti estaba a la sazón cautivo en Túnez ${ }^{59}$. En efecto, G.B. Vecchietti y su hermano Girolamo fueron dos famosos viajeros y orientalistas italianos, implicados en distintas misiones y viajes al servicio del papado. Giovan Battista, en concreto, fue enviado por Gregorio XIII como embajador a Persia en un viaje que se prolongó entre 1585-8. Realizó varios viajes por Oriente y Europa, y en 1608 estuvo unos meses cautivo en Túnez ${ }^{60}$.

Otra de las personas que aparece mencionada en el citado documento es «il sig.or Diego Perez de Mesa nativo di Ronda cità in Spagna. È filosofo mathematico et theologo. È stato cathedrante delle Mathematiche della Università di Alcalà et di Salamanca. Sà la lingua Arabica et la latina. Resiede in Roma [...]» ${ }^{61}$. La figura de Diego Pérez de Mesa es asimismo bien conocida: nacido en Ronda el 17 de diciembre de 1563, fue catedrático de matemáticas en la Universidad de Alcalá, y después, a partir de 1591, en la de Salamanca. Además de su obra propiamente científica, Pérez de Mesa fue autor de una Politica o Razón de Estado sacada de Aristóteles ${ }^{62}$, y de una recreación

58 Archivo de la Abadía del Sacromonte, Legajo VI, f. 1191.

59 Ibid.

60 V. Tucci, Ugo, «Una relazione di Giovan Battista Vecchietti sulla Persia e su Regno di Hormuz (1587)», Oriente Moderno, 35 (1955), 149-160; Richard, F. «Les manuscrits persans rapportés par les frères Vecchietti et conservés aujourd'hui à la Bibliothèque Nationale», Studia Iranica, 9 (1980), 291-300; Almagià, R., «Giovan Battista e Gerolamo Vecchietti viaggiatori in Oriente», Atti della Accademia Nazionale dei Lincei. Rendiconti. Classe di scienze morali, storiche e filologiche, Serie VIII, 11 (1956), 313-350. Sobre la importancia de Girolamo Vecchietti en las relaciones entre Roma y la iglesia copta, y su papel en la llegada a Italia de una gran cantidad de manuscritos coptos, v. Levi della Vida, G., Ricerche, 267 y passim.

${ }^{61}$ Archivo de la Abadía del Sacromonte, Legajo VI, f. 1191.

62 Pérez de Mesa, Diego, Política o razón de estado, ed. Pereña, L. y Baciero, C., 
del Libro de grandezas y cosas notables de España de Pedro de Medina ${ }^{63}$.

Entre los personajes citados en esa lista de posibles traductores de los Plomos, me ha sido posible situar también, aunque de manera más difusa, a «il sig. or Luis Felix di Seviglia. Ha qualque cognitione di Logica, filosofia et theologia, sa bene la lingua Arabica et la latina, ha servito la sedia apostolica nelle missione occorenti et in particolare nella missione fatta al Re di Persia da Papa Clemente VIII» ${ }^{64}$. En efecto, en la documentación de la embajada a Persia que, por orden de Clemente VIII, llevaron a cabo Diego de Miranda y Francisco da Costa, aparece mencionado un español llamado Luigi Filis o Ludovico Felix ${ }^{65}$. Estas alusiones son tan escuetas que apenas es posible sacar ninguna información adicional sobre su persona. Sí podemos adivinar, sin embargo, la posición de Félix en medio de las tremebundas disputas que enfrentaron a los dos embajadores en el curso de su tan desastrosa como apasionante embajada, que pasó por Praga, Varsovia, Astracán, Isfahan... Sabemos que, en 1602, Luis Félix formaba parte de los criados de Diego de Miranda que se pasaron al servicio del padre Francisco da Costa tras un gravísimo enfrentamiento entre ambos en Astracán a propósito de un muchacho valón que formaba parte de la expedición. No sabemos si continuó al servicio de éste, o si volvió con Miranda cuando, tras su estancia en Persia, los dos embajadores se separaron: Diego de Miranda regresó vía Ormuz y Goa, y llegó a Lisboa en 1604. El padre Costa, por su parte, regresó por Rusia acompañado de un embajador persa, se demoró varios años en Astracán, y no llegó a Roma hasta 1609. Luis Félix, por su parte, estaba ya en Roma en 1608, y, como muestra el documento que estoy uti-

con la colaboración de Abril, V., García, A. y Maseda, F., Madrid, 1980. Tomo los datos biográficos de Pérez de Mesa del estudio introductorio de esta edición.

${ }_{63}$ Pérez de Mesa, Diego, Primera y segunda parte de la grandeza y cosas notables de España [...]. Alcalá de Henares, 1595. La obra de Pedro de Medina está editada por González Palencia, Á., Obras de Pedro de Medina, Madrid, 1944. En la obra de Pérez de Mesa puede encontrarse, por ejemplo, un método muy detallado para calcular las correspondencias entre los años de la hégira y los de la era cristiana, al tiempo que una crítica a las «reducciones» efectuadas por Ambrosio de Morales y Luis de Mármol (v. Primera parte, cap. XVII)

${ }^{64}$ Archivo de la Abadía del Sacromonte, Legajo VI, f. 1191.

65 Alonso, Carlos, «Una embajada de Clemente VIII a Persia (1600-1609)», Archivum Historiae Pontificiae, 34 (1996), 7-25, y en especial 33, 47 y 113, donde aparece mencionado Luis Félix. 
lizando, su nombre apareció entre los posibles candidatos a traducir los Plomos.

En dicho documento se citan también, como era de esperar, varios miembros del Colegio Maronita de Roma, que había sido fundado en 1584, y cuya extraordinaria función como vía de transmisión de la cultura de los cristianos orientales a Europa es bien conocida. Uno de estos maronitas era "Gioanne Cipriotto della natione di Marroniti alunno del Collegio di Marroniti en Roma. Sta hoggi nel'isola di Cipro do la sua seconda moglie et sua casa». Al parecer, Gioanne había estudiado Lógica, Filosofia y Teología durante 18 años en el Colegio de Roma, y sabía «la lingua Arabica, la Chaldea, la Greca et la Latina» ${ }^{66}$. Creo que es posible identificar a este personaje con Yūhannā b. Ŷiryis, uno de los ocho primeros estudiantes maronitas que llegaron a Roma el 14 de diciembre de 1583 acompañando al padre Yūḥannā Ayyūb al-Ḥașrūnī. Yūhannā b. Ŷiryis era chipriota, de Vuno; a su llegada a Roma, hablaba el griego vulgar, y luego estudió latín, italiano, árabe y siriaco ${ }^{67}$. En nuestro documento también se hace mención de un «Sr. Giovani del Collegio di Marroniti in Roma, dove sono alcuni intelligenti della lingua Caldaica, Arabica et Latina, et sono disciplinati», quien, de manera más conjetural que en el caso anterior, podría ser identificado con el famoso Juan Hesronita, que estudió en Roma entre 1603 y 1614, año en que viajó a París junto con el célebre Gabriel Sionita ${ }^{6}$.

Finalmente, y además del nombre bien conocido de Raimondi, nuestro documento se refiere a otros personajes que no he podido identificar con precisión: uno de ellos es «messer Theodolo di natione Tartaro, era Mahomettano, et hoggi per gratia de Dio è Christiano, battezzato et cathechizato in Venetia, resiede in Roma, et ha qualque trattenimento da Gio. Btt. ${ }^{a}$ Raimondo, qual ha la suprema cura et intelligentia del negotio delle lingue straniere; sà il detto Theodolo la lingua Persiana sua nativa, la lingua Arabica, imparata do studio et fati-

66 Archivo de la Abadía del Sacromonte, Legajo VI, f. 1191.

67 V. Gemayel, Nasser, Les échanges culturels entre les maronites et l'Europe. Du Collège de Rome (1584) au Collège de 'Ayn-Warqa (1789), 2 vols., Beyrouth, 1984, I, 34 y 97; Harfūšs, Ibrāhīm, "Talāmidat al-madrasa al-rūmāniyya al-mārūniyya al-qadīma», al-Manāra, 6 (1935), 661-673, 662 y 664 [este artículo continúa en las pp. 733-745, 802-812 y VII (1936) 18-27, 184-187, 258-273, 424-432, 543-550].

${ }^{68}$ Raphael, Pierre, Le rôle du Collège maronite Romain dans l'orientalisme au XVIIe et XVIIIe siècles, Beyrouth, 1950, 96. 
ca sua, la lingua torchesca et qual poco della lingua indiana». El otro

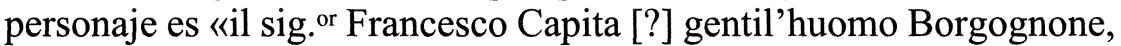
et canonico in Bisinzone, theologo et filosofo. Ha cognitione piu che ordinaria della lingua Arabica, Hebraica, Ethiopica, Greca et Latina», a quien no he podido identificar, quizás por una mala lectura de su apellido. En todo caso, y como queda dicho, quien finalmente viajó a España para trabajar en la traducción de los Plomos fue Marcos Dobelio, a quien el documento sacromontano se refiere como «lecâtino della natione di Gurdi resiede in Roma, et ha la Cathedra in Roma della lingua Arabica, et sa anco la latina»; aunque tampoco debería olvidarse, para completar el cuadro de las relaciones del Sacromonte con los arabistas de Italia, la figura de Sergio Maronita o Sarkīs al-Šadrāwī. Debemos analizar aún, sin embargo, con el detalle que merece, el auténtico alcance de las pesquisas de d. Pedro de Castro en busca de traductores fiables del árabe, y las interesantísimas conexiones que dicha búsqueda produjo con algunas partes de Europa y el Norte de África.

La vida y el destino itinerante de personas como Diego de Urrea o Marcos Dobelio no son enteramente azarosos, y nos ayudan a conectar el episodio de los Plomos con el gran proceso de formación del saber orientalista en la Europa moderna. Los suyos son buenos ejemplos de biografías que, cruzadas o paralelas, desarrolladas en torno al conocimiento del árabe y la traducción de documentos y libros en esa lengua, se encuentran en episodios comunes que convocan el afán bibliófilo, el interés político y religioso o la inquietud científica. Una mirada más detallada sobre esta historia nos permitirá discernir las diferentes implicaciones que el desarrollo y afianzamiento del arabismo tuvo en lugares distintos como España e Italia. La simple comparación entre don Pedro de Castro y Federico Cesi ilustra esas diferencias, que atañen a cuestiones como, por ejemplo, la traducción y edición de textos orientales. Si los últimos años de la vida de Diego de Urrea en Italia nos conducen hasta Galileo o Pietro della Valle, la historia de los Plomos evoca el origen de los españoles y su lengua, como muestran los números monográficos que esta revista ha dedicado a la cuestión. Esto explica, en buena medida, la singularidad de este primigenio «orientalismo» español, y los avatares de su evolución. 


\title{
RESUMEN
}

Este artículo trata de la estancia en Italia del traductor del árabe Diego de Urrea, que pasó los últimos años de su vida en Nápoles. Su relación con círculos eruditos italianos, como el del príncipe Federico Cesi y su Accademia dei Lincei, a la que perteneció Galileo, pone de relieve algunos de los rasgos característicos del «orientalismo» italiano de la época, y sus relaciones y diferencias con lo que ocurría en España a comienzos de s. XVII, cuando el asunto de los Plomos del Sacromonte ejerció un papel fundamental en la definición de la lengua y la historia árabes dentro de la historia de España.

\begin{abstract}
This paper deals with the Italian sojourn of Diego de Urrea, who spent the last years of his life in Naples. His relationship with the Italian learned milieu, especially with Prince Federico Cesi and his Accademia dei Lincei, of which Galileo Galilei was a member, sheds some light on the traits of the Italian "orientalism" at that time, and its coincidences and differences with what happened in Spain at the beginning of the 17th Century, when the affaire of the "Lead Books of the Sacromonte" was an essential factor in the way in which Arabic and the Islamic past was to be incorporated in the history of Spain.
\end{abstract}

